\title{
Energy efficient software and hardware configuration of the digital substation in accordance with IEC 61850
}

\author{
Wisam Al-Tibbi* \\ Don State Technical University, Industrial automation department, 344000 Rostov-On-Don, \\ Gagarin sq. 1, Russia
}

\begin{abstract}
The features of configuring software and hardware of modern data acquisition systems as part of a digital substation to comply with the international standard IEC 61850 are considered. Given recommendations for energy efficient software and hardware configuration for $110 \mathrm{kV}, 6-35$ $\mathrm{kV}$ and $0.4 \mathrm{kV}$ power consumption level which allow you to increase the reliability of the system by $1.5-2$ times.
\end{abstract}

\section{Introduction}

A modern electrical substation is designed for distribution and conversion of electric power and combines both standard electrical devices of relay protection and power metering, as well as electrical automation and control devices. As the number of consumers and the number of recorded parameters is constantly growing, the number of simultaneously transmitted signals and data transmission channels is also increasing. All this data needs continuous processing and analysis in modern ERP and SCADA systems. The main requirement for such a digital substation $[1,20]$ is data processing in real time without the constant presence of supervisory personnel. The efficient transmission and redistribution of energy through smart grids has a direct impact on improving the energy efficiency of the system as a whole

To program and operate such automation systems it is necessary to use clear standards and data transfer technologies. At the software level, such standards include the IEC 61850 standard $[2,11]$, which describes the data transfer model.

\section{Digital substations structure}

The key hardware elements of a digital substation system (DSS) operating in accordance with this standard include: digital current and voltage transformers; analog and digital multiplexer units (Merging Units); Micro RTUs; intelligent electronic devices (IEDs).

Modern data transfer protocols that ensure the interconnection of hardware and software systems are vertical (MMS) and horizontal (GOOSE) exchange protocols [3,4].

\footnotetext{
* Corresponding author: nb1979@mail.ru
} 
The information that is transferred between the process layer and the network layer, as well as between the various other devices in the network layer, is only transferred using the fieldbus. Data exchange at the substation level, as well as from network level devices to substation level devices, also takes place using the substation bus $[5,17]$.

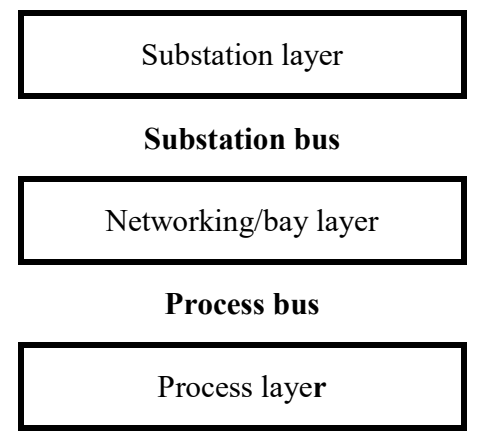

Fig. 1. Digital substation structure

The 1st layer of the process combines mainly the information from all power devices, namely from their control units. At this stage, the interfacing of the main equipment of the DSS is organized. Discrete information is collected from the main equipment contacts and digitized. Analog information is also collected and digitized. After that all collected information is transmitted to the next layer, from which the control command in digital form with the effect on the main equipment (for example, switching on / off the switching device) can be obtained.

The 2nd bay/network level includes equipment, which includes microprocessor terminals of relay protection, control and interlocking systems. Devices which receive data from the process level equipment itself, issue their commands to control the switchgear, and they also have information communication with the substation level equipment.

The 3rd layer (i.e. substation layer) is the automated workplaces of the personnel who control the substation equipment. Their places include control panels, mnemonic schemes, supervisory control and data collection systems. This layer also contains information about the state of the equipment itself and modes of operation. The worker processes the necessary information and transmits it to the higher level.

\section{Digital information exchange method}

Let's consider the digital exchange organization method example at control system, implemented in accordance with IEC 61850 standard. On the example of the structure shown in Figure 2 the interface in the SCADA-system is implemented. The elements of the structure are selected depending on the standardized levels of energy consumption $[6,18]$.

Depending on the level of power consumption, the following blocks can be distinguished on the block diagram:

$110 \mathrm{kV}$ transformer and generator line control cabinets, made in an industrial design, that is, a standard panel as required by the panel, in which two control and protection terminals are installed each. Exchange of information between these devices is carried out according to IEC 61850 protocol, which allows the system to function in accordance with the requirements of the protocol. 


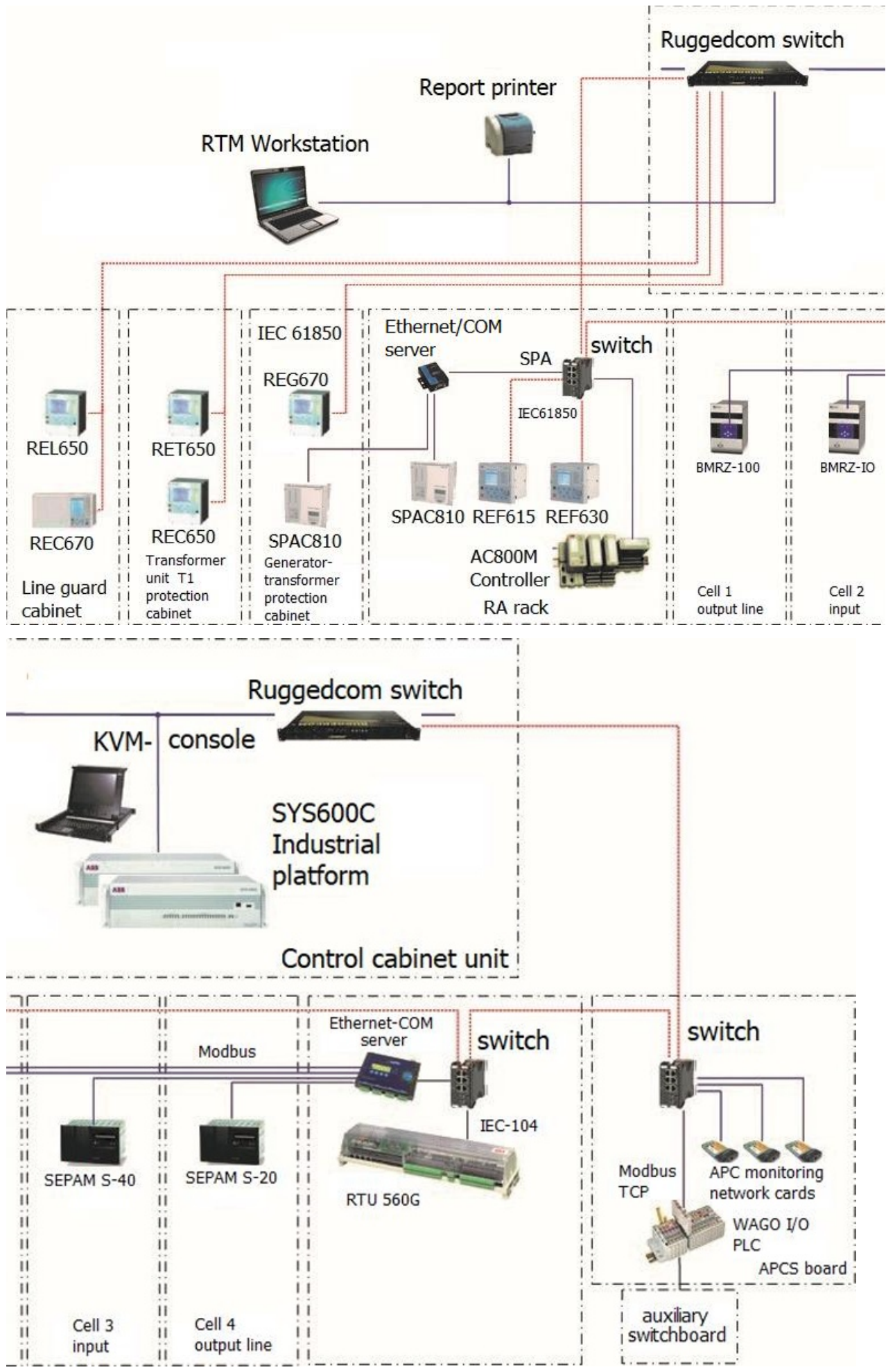

Fig. 2. Digital control system, implemented in accordance with IEC 61850 standard [6] 
The 6-35 kV switchgear is a device consisting of two input cabinets and two power line cabinets. These cabinets are equipped with terminals from different manufacturers, which will help to understand not only the connection diagrams, but also to see the differences between the terminals and their common features.

The auxiliary panel and the DC panel are also represented by real switchgear. And in one of the cabinets there is a data acquisition controller for $0.4 \mathrm{kV}$ and a DC panel, which also provides information exchange.

The elements of the structure are chosen depending on the standardized levels of energy consumption. Depending on the level of power consumption, the following blocks can be distinguished in the structural diagram.

Control cabinets of $110 \mathrm{kV}$ transformer and generator lines, made in industrial design, i.e. a standard panel according to panel requirements, in which two control and protection terminals are installed each. Exchange of information between these devices is carried out according to IEC 61850 protocol, which allows the system to function in accordance with the requirements of the protocol.

The $6-35 \mathrm{kV}$ switchgear is a device consisting of two input cabinets and two power line cabinets. These cabinets are equipped with terminals from different manufacturers, which will help to understand not only the connection diagrams, but also to see the differences between the terminals and their common features.

The auxiliary panel and the DC panel are also represented by real switchgear. And in one of the cabinets there is a data acquisition controller for $0.4 \mathrm{kV}$ and a DC panel, which also provides information exchange.

\section{Hardware configuration (IEC 61850 protocol settings)}

Work on any top-level IEC 61850 project starts with adding necessary elements to SCADA. In the system tree the elements are created in which the physical execution of the developed project takes place - automated workstations, controllers, cloud services, etc. For example, in the Master SCADA 4D environment [7,13] these elements are called Nodes. The system tree defines the list of used nodes and their settings from the number of supported nodes.

Each node contains its own groups. The ARMs include: Graphical Interface, Services (provide links between different actuation systems included in the same project, as well as saving data that is later used for hot restarts), Archives, Tasks, External Channels, Protocols, etc. For SCADA to act as a client, it is necessary to add the IEC61850 protocol to the Protocols node group (Fig.3).

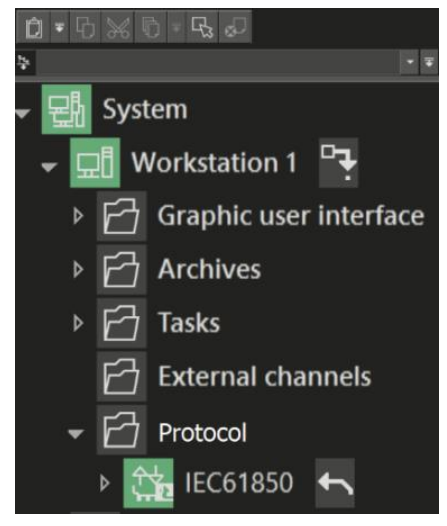

Fig. 3. Adding the IEC61850 protocol to the system tree 
In order to add channels, it is necessary to connect to the server (let's take REL 650 IED as an example - Fig.4).

We see that the parameters for REL 650 are: phase operating current; zero sequence operating current; arc protection; current protection; earth fault protection. The list of channels and their settings are formed automatically. No additional channel settings are required. When you first add a protocol, the settings are also formed automatically, but if necessary, you can change them in the "properties panel".

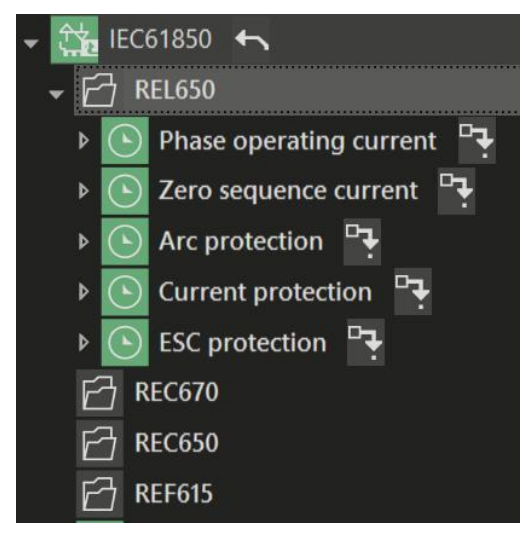

Fig. 4. IEC61850 protocol settings at SCADA system

\section{Screens Development}

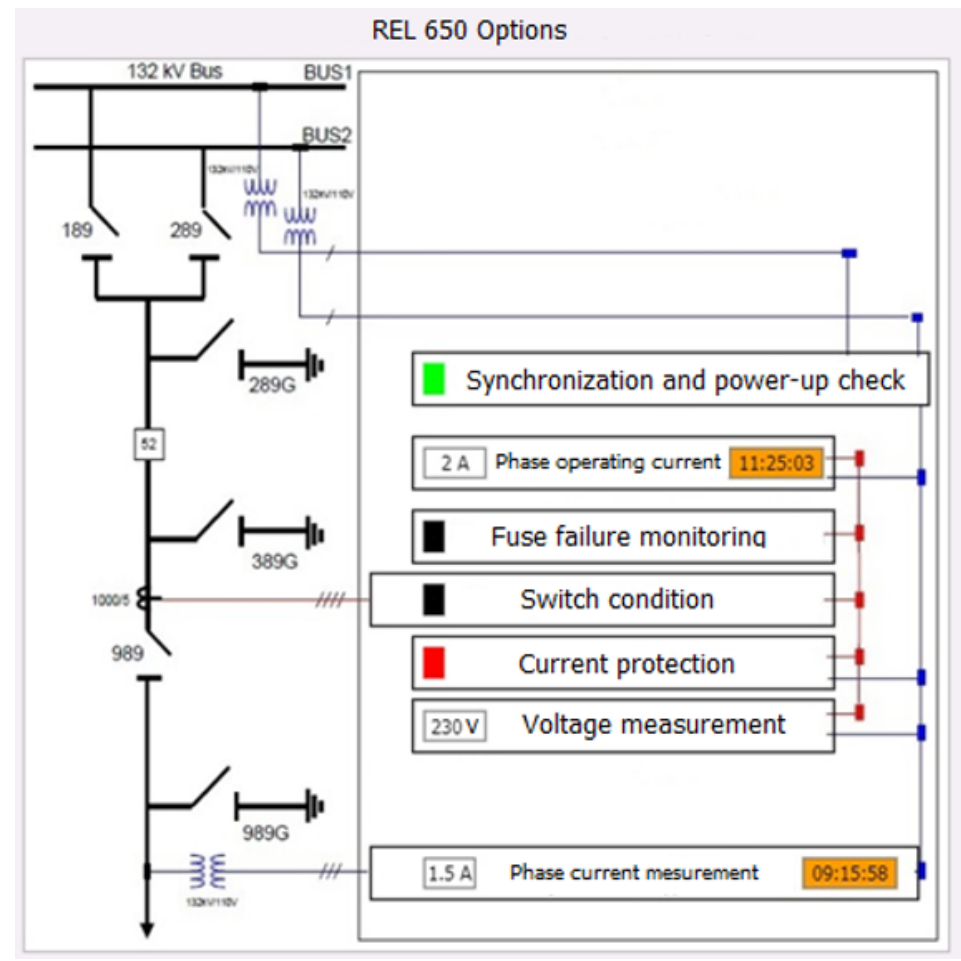

Fig. 5. REL 650 IED options screen 
The next stage of development is the "Graphical interface". In it we will develop both the main screen and additional screens. For example, let's create a diagram of an intelligent electronic device REL 650 (Fig.5), and see what parameters are present and working at a certain moment.

Such additional screens are connected to the upper level directly via the IEC 61850 protocol. But there is also industrial equipment that does not support this standard and communicates via the generally applicable industrial protocols Modbus, ProfiBus, etc. In this case we can, for example, use OPC technology.

\section{Using the OPC server to communicate with the upper level}

The advantage of this technology is that, firstly, it provides connection of heterogeneous nodes within the project and connection to other hardware and software systems, and the user does not need to manually assign communication lines and exchange protocols. Any node in the system tree can become an OPC-server and transmit data to clients.

Secondly, not every SCADA-system supports the IEC 61850 standard, and OPCtechnology will help in this. Let's consider as an example the configuration of OPC-server IEC 61850 Master OPC Server [8] designed to work with any equipment that supports data exchange via protocol described in IEC-61850 standard. The server is implemented as a plug-in for Multi-Protocol Master OPC Server [9].

The main advantages of the OPC server include high performance, ease of installation and use. It minimizes disruptions and failures. This guarantees stable operation and uninterrupted data collection. The program is most often used for automation and dispatching of high-voltage substations. Let's configure the OPC-server in the above program (Fig.6).

We will add the IEC 61850 protocol to the "Server" object and then the "GOOSE" device. The principle of GOOSE operation is based on sending broadcast telegrams to a wide range of recipients. The settings relevant to the controller are under "Protocol Properties". Where, "network adapter-receiver" is the identifier of the network adapter to which GOOSE packets arrive; "Control Unit" is the text identifier of the control unit. "Numeric identifier" is the numeric identifier of the control block.

In order to get the data written to the controller, a "Protocol Tag" is needed. The tag is added via the context menu of the device or group. The basic IEC61850 protocol tag settings are: "MMS Data Type" is the data type of the parameters being polled. "Number in the control block" is the number of the variable in the received control block.

In accordance with the developed system, let's add the necessary IEUs with their parameters.

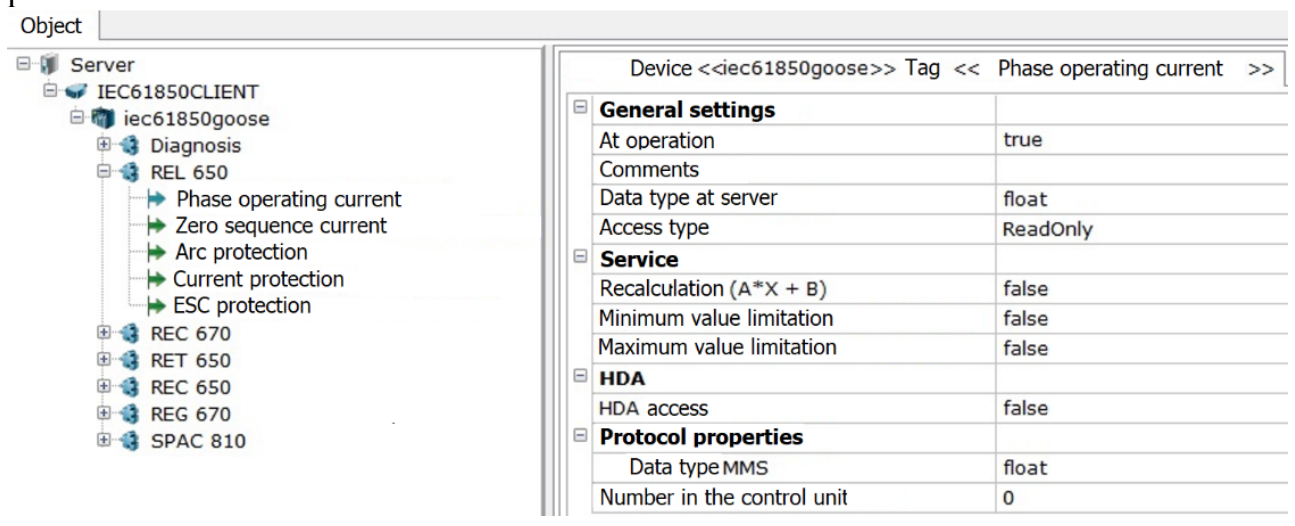

Fig. 6. IEU with parameters in the OPC-server 
Next, to see the properties, simply click on the desired parameter.

\section{Summary}

In this paper we have considered the features of control and data acquisition systems according to the IEC 61850 standard. Implementation of the IEC 61850 standard makes it possible to connect all process equipment in a substation with a single information network, which transmits not only data from measuring devices to relay terminals, but also control signals. Application of GOOSE protocol (included in IEC 61850 standard) in combination with correct design and parameterization of information network and relay protection devices allows to refuse from using copper circuits for signals transfer, providing the required level of reliability and performance. In other words, by using protocol, all signals between terminals are transmitted over a single (e.g., optical) cable. The use of a single cable instead of conventional signal cables reduces the duration and cost of substation downtime during the reconstruction of secondary equipment and makes it possible to easily and quickly reconfigure the system. Thus, the exchange between cabinets is carried out only through a digital channel, which eliminates the need for traditional circuits connecting the cabinets. Data using this standard is transmitted to the SCADA system in a unified form. SCADA system, in its turn, acts as a database, where the information is presented in the form of mnemonic diagrams, graphs, etc. (there is no need for manual calculations).

The energy efficient transmission and redistribution of energy through smart grids has a direct impact on improving the energy efficiency of the system as a whole. The systems considered allow increasing the reliability of the system by $1.5-2$ times $[10,20]$. The effect of equipping the dispatcher room with network control tools is to reduce the amount of power shortfalls and the duration of power outages due to:

- Analysis of responsiveness and selectivity of digital protections;

- Rapid identification of fault locations, localization of emergency areas and restoration of power supply;

- Operational management of field teams;

- Reducing the time for preparation, execution and closing of work orders and instructions;

- Multiple use of switching forms;

- Reduction of transportation costs by $15 \%$ or more and reduction of power losses by $10 \%$ or more.

\section{References}

1. S. Meier, T. Werner, Performance considerations in digital substation applications, in Proceedings of the 13th International Conference on Development in Power System Protection 2016 (DPSP), DOI:10.1049/cp.2016.0057, (2016).

2. IEC 61850:2021 SER, Communication networks and systems for power utility automation - ALL PARTS, (2021).

3. C. Kriger, S. Behardien, John-Charly Retonda-Modiya, A Detailed Analysis of the GOOSE Message Structure in an IEC 61850 Standard-Based Substation Automation System, Computer Science Int. J. Comput. Commun. Control, DOI:10.15837/IJCCC.2013.5.329, (2013).

4. R. Mackiewicz, Technical Overview of IEC 61850 and benefits, Power Systems Conference and Exposition, PSCE '06. 2006 IEEE PES, DOI:10.1109/PSCE.2006.296392, (2006). 
5. R. Miller, Industrial Electricity and Motor Controls, Second Edition, 2013, ISBN-10: 0071818693, (2013)

6. A. Trofimov, A. Polyakov, A. Maslov, Experience of use of the training complex ACS electrical equipment of power stations and substations, Automation and IT in the energy sector J. (ISSN: 2410-4043), 5 (2017).

7. Master SCADA 4D (INSAT website), Available at:

https://insat.ru/products/?category=1536, Accessed: May 14, (2021).

8. Modbus Universal Master OPC Server (INSAT website), Available at: https://insat.ru/products/?category=1414, Accessed: May 14, (2021).

9. Multi-Protocol Master OPC Server (INSAT website), Available at: https://insat.ru/products/?category=4004, Accessed: May 14, (2021).

10. ABB Power and Automation Systems Energy Efficiency Technology. Smart Grid. (ABB library website), Available at: https://library.e.abb.com/public/79329f1dcc4fe463482578a40030840d/energy_efficien cy 6.pdf, Accessed: May 14, (2021).

11. H. Dawidczak, IEC 61850 - Erfahrungen aus kundenprojekten und interoperabilitä tstests, ETZ, Elektrotechnik und automation, 6 (2006).

12. L. Yang., P. Crossley, et al., Grasset H.4 protection performance testing in IEC 61850 based systems, 10th IET International Conference on Developments in Power System Protection, DPSP, (2010).

13. M.R. Silva, P. Machado, et al., Modelling using colored petri net of communication networks based on IEC 61850 in a microgrid context, Journal of control, automation and electrical systems, 6 (2018).

14. J.H. Hong, D. Ishchenko, et al., Implementation of Resilient Self-Healing Microgrids with IEC 61850-Based Communications, ENERGIES, DOI: 10.3390/en14030547, 14 (2021).

15. F. Diaz, F. Guerrero, et al., Technical-economic evaluation model for a process bus based on IEC 61850, Sustainable energy grids \& networks, DOI: 10.1016/j.segan.2019.100288, 21 (2020).

16. T. Ustun, M. Aftab, et al., A Novel Scheme for Performance Evaluation of an IEC 61850-Based Active Distribution System Substation, IEEE ACCESS, DOI: 10.1109/ACCESS.2019.2937971, 7 (2019).

17. S. Gupta, A. Gandhar, et al., Reliability analysis of a novel IEC 61850-9-2 process-bus based substation automation system architecture, Journal of statistics \& management systems, 23 (2020).

18. N. Ali, M. Eissa, et al., Accelerating the protection schemes through IEC 61850 protocols, International journal of electrical power \& energy systems, DOI: 10.1016/j.ijepes.2018.04.035, 102 (2018).

19. M. T. Rashid, S. Yussof, et al., Trust System Architecture for Securing GOOSE Communication in IEC 61850 Substation Network, International journal of security and its applications, DOI: 10.14257/ijsia.2016.10.4.27, 10 (2016).

20. Y. Cai, Z. Cai, et al., Communication-Assisted Protection and Self-Healing Control Scheme for Distribution Networks Based on IEC 61850, IEEE ACCESS, DOI: 10.1109/ACCESS.2020.2987921, 8 (2020). 